\title{
Prática baseada em evidencia: um levantamento em profissionais atuantes na área de fisioterapia hospitalar: um estudo transversal
}

\author{
Evidence-based practice: a survey of working professional in the hospital physiotherapy \\ area: a cross-sectional study
}

Pâmela Dutra Collato Costa ${ }^{1}$

Tatiane Regina Sousa ${ }^{2}$

Renato Claudino ${ }^{3}$

Autor correspondente:

Pâmela Dutra Collato Costa,

Rua Santo Antônio, 968, apto 202 B.

Barreiros, São José, SC, Brasil, 88117-351.

pamelacollato@hotmail.com

Fisioterapeuta do Hospital Regional de São José Dr. Homero de Miranda Gomes.

Aluna do curso de pós-graduação em Fisioterapia Intensiva. Centro Universitário Estácio de Santa Catarina, São José, Brasil.

pamelacolatto@hotmail.com

Fisioterapeuta, Mestre em Fisioterapia - UDESC CEFID.

tatianereginafisio@gmail.com

Fisioterapeuta, Mestre em Ciências do Movimento Humano - UDESC - CEFID

renato.claudino@sc.senac.br

\begin{abstract}
Resumo
Introdução: A Prática Baseada em Evidências (PBE) consiste em uma combinação entre a experiência do terapeuta, a melhor evidência disponível e a preferência/adaptação do paciente. O fisioterapeuta hospitalar detém conhecimentos sobre procedimentos terapêuticos validados por pesquisas, com comprovação da eficácia de suas técnicas. Sua presença é indispensável em setores como UTI adulto, UTI neonatal e enfermarias. Por se tratar de uma área primordial para a reabilitação de pacientes internados, faz-se necessário identificar se os profissionais, que atuam nos diversos setores do ambiente hospitalar, estão devidamente aptos a oferecer o melhor serviço disponível utilizando os princípios da PBE.

Objetivo: Descrever comportamentos, conhecimentos, habilidades, opiniões e barreiras relacionados à PBE reportados por fisioterapeutas hospitalares atuantes em um hospital público estadual de Santa Catarina.

Métodos: Foi utilizado um questionário eletrônico adaptado que identificou comportamentos, conhecimentos, habilidades, opiniões e barreiras em PBE reportados pelos participantes.

Resultados: A taxa de resposta ao questionário foi de $72,72 \%$ (16 respondentes). O meio de atualização profissional mais utilizado foram os artigos científicos (56,3\%), sendo a base mais acessada a Scielo $(93,8 \%)$ seguida da Pubmed $(81,3 \%)$. Os fisioterapeutas $(87,5 \%)$ relatam que sabem o significado do termo PBE e procuram implantar a melhor evidência científica na prática clínica $(75,0 \%)$, auxiliando na qualidade dos serviços de saúde $(56,3 \%)$. As barreiras mais relatadas foram o idioma dos artigos, a falta da qualidade de evidências, a dificuldade de obter o artigo na íntegra, a falta de tempo, a dificuldade de entendimento dos dados estatísticos, a interpretação dos resultados e a falta de treinamento em PBE.

Conclusão: Os fisioterapeutas hospitalares relatam atitudes positivas quanto ao uso da PBE, apresentam interesse em aprofundar seus conhecimentos sobre o tema a fim de utilizar a melhor evidência científica na prática clínica, visando à qualidade no atendimento ao paciente. Por outro lado, algumas barreiras dificultam a implantação da PBE na rotina diária de atendimento.
\end{abstract}

Descritores: Prática clínica baseada em evidencias. Fisioterapia. Ambiente hospitalar.

\begin{abstract}
Introduction: The Evidence-Based Practice (EBP) consists of a combination of the therapist's experience, the best evidence available and the patient's preference / adaptation. The hospital physiotherapist possesses knowledge on therapeutic procedures validated by researches with proof of the effectiveness of his techniques. His/her presence is indispensable in sectors such as adult ICU, neonatal ICU, and infirmaries. Because it is a primary area for the rehabilitation of hospitalized patients, it is necessary to identify whether the professionals, who work in the various sectors of the hospital environment, are able to offer the best available service using the principles of the PBE.

Objective: To describe the behavior, knowledge, abilities, opinions and self-reported barriers related to PBE of hospital physioterapists at a public state hospital in Santa Catarina, Brazil.

Methods: An adapted electronic questionnaire was used that identified behavior, knowledge, skills, opinions and barriers in self-reported EBP by participants.

Results: The response rate to the questionnaire was $72.72 \%$ (16 respondents). The most used professional updating medium were scientific articles $(56.3 \%)$, the most accessed basis was Scielo $(93.8 \%)$, followed by Pubmed (81,3\%). Physical therapists $(87,5 \%)$ report that they know the meaning of the term PBE, seek to implant the best scientific evidence in clinical practice $(75,0 \%)$, thus helping the quality of health services $(56,3 \%)$. The most reported barriers were the language of the articles, lack of quality of evidence, difficulty in obtaining the full article, lack of time, difficulty in understanding statistical data, interpretation of results, and lack of training in PBE.

Conclusion: Hospital physioterapists report positive attitudes regarding the use of EBP, are interested in deepening their knowledge about the subject in order to use the best scientific evidence in clinical practice aiming at quality in patient care. On the other hand, some barriers make it difficult to implement EBP in the daily care routine.
\end{abstract}

Keywords: Evidence-based practice. Physical therapy. Hospital environment.

\section{Cite como \\ Vancouver}

Costa PDC, Sousa TR, Claudino R. Prática baseada em evidencia: um levantamento em profissionais atuantes na área de fisioterapia hospitalar: um estudo transversal. Conscientiae Saúde 2019 out./dez.;18(4):414-428. https://doi.org/10.5585/conssaude.v18n4.14364. 


\section{Introdução}

A Prática Baseada em Evidências (PBE) surgiu na década dos anos 90 e consiste em uma combinação entre a experiência do terapeuta, a melhor evidência disponível e a preferência/adaptação do paciente ${ }^{1}$. A utilização da PBE é considerada necessária e importante por fisioterapeutas, sendo que os profissionais se baseiam nas evidências publicadas para apoiar a tomada de decisão do plano de tratamento ${ }^{2,3}$.

Para adotar a PBE na rotina de atendimentos, o profissional deve dispor da melhor evidência publicada para tratar problemas clínicos, baseando-se nestes princípios:

1) formulação de uma questão clínica;

2) busca na literatura;

3) avaliação crítica da validade da evidência;

4) aplicação dos resultados da evidência na prática clínica; e

5) avaliação dos efeitos da implementação ${ }^{1,2}$.

A PBE torna-se um conjunto indispensável de interpretar informações e tem-se tornado, na prática clínica, importante apoio na tomada de decisão para a maioria dos profissionais da saúde. Em alguns países, é tomada como um pré-requisito para processos de acreditação e indicador de qualidade na prestação de saúde em fisioterapia 10,12,13, 14, 15,16.

Em uma revisão sistemática relativamente às barreiras identificadas pelos fisioterapeutas para implementação da PBE, verificou-se que as principais foram: incapacidade de interpretação de dados estatísticos; falta de suporte do empregador; falta de tempo; falta de recursos; falta de interesse e falta de generalização dos resultados dos estudos (dificuldades no entendimento dos resultados bem como nas análises estatísticas) ${ }^{3}$. Além disso, a dificuldade em relação ao domínio do idioma, principalmente da língua inglesa, também pode ser considerada um obstáculo ${ }^{1,3}$.

Outras barreiras mencionadas pelos participantes são problemas relacionados às políticas de saúde vigentes, a complexidade da prática fisioterapêutica, a capacidade do fisioterapeuta de aplicar com competência a melhor intervenção baseada nas evidências, fatores socioeconômicos e culturais, assim como dificuldade de acesso a textos completos e a programas de educação continuada. Essas adversidades são encontradas nas mais diversas áreas de atuação fisioterapêutica ${ }^{4,5}$.

Na década de 1970, reconheceu-se a relevância da fisioterapia hospitalar, especialmente na área respiratória na assistência. Na década seguinte, houve crescimento da especialidade 
quando essa começou a compor a equipe multiprofissional nas unidades de terapia intensiva: UTI adulto, UTI neonatal e enfermarias ${ }^{6,8}$.

A Resolução n. ${ }^{\circ}$ 402/2011 do Conselho Federal de Fisioterapia e Terapia Ocupacional (COFFITO) dispõe que a presença do fisioterapeuta na assistência hospitalar traz benefícios não somente ao paciente - menor risco de infecção hospitalar, menor tempo de internação como também redução dos gastos e diminuição das complicações ocasionadas pelo imobilismo no leito a partir da mobilização precoce, fortalecimento muscular e uso de exercícios estacionários ${ }^{7,9}$. Entretanto, enquanto na área da fisioterapia hospitalar há estudos que envolvem recomendações para adoção do modelo PBE, sua percepção na prática clínica ainda é escassa ${ }^{20,21}$.

Por se tratar de uma área primordial para a reabilitação de pacientes internados, faz-se necessário identificar se os profissionais que atuam nos diversos setores do ambiente hospitalar utilizam os princípios da PBE. Desta forma, este estudo tem como objetivo descrever o comportamento, conhecimento, habilidades, opiniões e barreiras relacionadas à PBE reportados por fisioterapeutas hospitalares em um Hospital Público Estadual de Santa Catarina.

\section{Metodologia}

Tipo de estudo

O presente estudo caracteriza-se como descritivo exploratório e de caráter transversal. Foi aprovado pelo Comitê de Ética em Pesquisa do Instituto de Cardiologia de Santa Catarina, São José, SC (CAAE: 04826818.0.0000.0113).

\section{Participantes}

Foram critérios de inclusão para os participantes desta pesquisa: profissionais fisioterapeutas hospitalares, efetivos ou contratados pela Secretaria de Estado da Saúde de Santa Catarina (SES-SC) e atuantes no Hospital Dr. Homero de Miranda Gomes - Hospital Regional de São José (HRSJ), devidamente registrados no CREFITO-10.Instrumento de coleta

Aplicou-se aos participantes da pesquisa um questionário com perguntas relativas a comportamento, conhecimento, habilidades, opiniões e barreiras relacionadas à Prática Baseada em Evidências (PBE). A presente pesquisa baseia-se em estudo prévio de Silva e colaboradores, realizado com profissionais fisioterapeutas (5), com adaptação das perguntas à especialidade de fisioterapia hospitalar. O questionário compreendeu estas categorias: 
a) Dados demográficos e vivência acadêmica da graduação e atuação profissional (tempo de graduação, instituição onde obteve a graduação, nível de formação, setor em que trabalha, experiência ambulatorial e docência no ensino superior);

b) Área de atuação em fisioterapia cardiorrespiratória;

c) Habilidades com o idioma inglês;

d) Informações sobre PBE (comportamento, conhecimento prévio em recursos importantes em PBE, habilidades e recursos relacionados a PBE; opiniões sobre PBE, barreiras percebidas relacionadas a PBE).

O questionário continha apenas questões fechadas, com respostas de múltipla escolha, em escala do tipo Likert de cinco pontos $(1=$ discordo completamente, $2=$ discordo parcialmente, 3 = neutro, 4 = concordo parcialmente, $5=$ concordo completamente) (5).

\section{Procedimentos de coleta}

A pesquisadora principal enviou o questionário eletrônico para todos os fisioterapeutas do HRSJ via correio eletrônico (profissional e/ou pessoal) que se obteve mediante contato com a chefia do serviço de fisioterapia do referido hospital. O questionário eletrônico foi construído na ferramenta Jot Form ${ }^{\circledR}$ do navegador Google Crome ${ }^{\circledR}$. Trata-se de uma ferramenta de serviço gratuito para a construção de formulários online. Para divulgação e acesso ao questionário, utilizou-se o aplicativo Google Sites ${ }^{\circledR}$, que permitiu a criação de páginas eletrônicas na rede mundial de computadores.

\section{Análise dos dados}

Os dados foram analisados utilizando estatística descritiva, a partir do programa IBM SPSS $^{\circledR}$ versão 22.0 (IBM, Chicago, IL, EUA) e expressos em porcentagens e frequências.

\section{Resultados}

A taxa de resposta ao questionário do estudo foi de 72.72\% (16 respondentes). A Tabela 1 apresenta os dados demográficos dos participantes. A maioria dos participantes são do sexo feminino (93,8\%), com tempo de formação entre 10 e 14 anos, formados em instituição privada $(56,3 \%)$ e com pós-graduação lato sensu (62,5\%). Oito deles $(50 \%)$ declararam ter boa 
habilidade na leitura de textos em língua inglesa. Todos atuam com o atendimento ao paciente no ambiente hospitalar. Seis deles $(37,5 \%)$ têm experiência com docência. A área de maior interesse, além da fisioterapia hospitalar, é a cardiorrespiratória (43,8\%).

Tabela 1 - Características demográficas dos participantes (n 16)

\begin{tabular}{|c|c|}
\hline Características & n $(\%)$ \\
\hline \multicolumn{2}{|l|}{ Gênero } \\
\hline Masculino & $1(6,3)$ \\
\hline Feminino & $15(93,8)$ \\
\hline \multicolumn{2}{|l|}{ Tempo de formação } \\
\hline Menos que 5 anos & $1(16,1)$ \\
\hline 5- 9 anos & $2(20,5)$ \\
\hline $10-14$ anos & $3(26,8)$ \\
\hline 15-19 anos & $2(20,5)$ \\
\hline 20-24 anos & $0(0,0)$ \\
\hline Mais que 24 anos & $1(16,1)$ \\
\hline \multicolumn{2}{|l|}{ Nivel de formação atual } \\
\hline Graduação & $1(6,3)$ \\
\hline Pós-graduação Lato sensu & $10(62,5)$ \\
\hline Mestrado & $4(25,0)$ \\
\hline Doutorado & $1(6,3)$ \\
\hline \multicolumn{2}{|l|}{ Procedência da sua graduação } \\
\hline Privada & $9(56,3)$ \\
\hline Publica & $7(43,8)$ \\
\hline \multicolumn{2}{|l|}{ Exerce a profissão atualmente } \\
\hline Atendimento ao paciente & $16(100)$ \\
\hline \multicolumn{2}{|l|}{ Setor de trabalho como fisioterapeuta } \\
\hline Privado & $1(6,3)$ \\
\hline Não trabalho em outro setor além do hospital & $8(50,0)$ \\
\hline Profissional liberal (autônomo) & $7(43,8)$ \\
\hline \multicolumn{2}{|l|}{ Experiência em docência } \\
\hline Sim & $6(37,5)$ \\
\hline Não & $10(62,5)$ \\
\hline \multicolumn{2}{|c|}{ Qual outra área de interesse além da fisioterapia hospitalar } \\
\hline Fisioterapia no trabalho & $1(6,3)$ \\
\hline Cardiorrespiratória & $7(43,8)$ \\
\hline Traumatologia e ortopedia & $1(6,3)$ \\
\hline Neurofuncional & $5(31,3)$ \\
\hline Esportiva & $1(6,3)$ \\
\hline \multicolumn{2}{|l|}{ Habilidade na leitura de textos em inglês } \\
\hline Ruim & $2(12,5)$ \\
\hline Razoável & $6(37,5)$ \\
\hline Boa & $8(50,0)$ \\
\hline
\end{tabular}




\section{Comportamento em relação à $\mathrm{PBE}$}

A Tabela 2 descreve o comportamento dos fisioterapeutas hospitalares em relação à PBE. O meio de atualização profissional mais utilizado são os artigos científicos (56,3\%), sendo que $25 \%$ dos participantes apontaram acessar as bases de dados de uma a três vezes por mês. As bases já utilizadas foram Scielo, Pubmed e Lilacs (93,8\%), sendo que a base mais acessada foi a Scielo $(93,8 \%)$, seguida da Pubmed (81,3\%), geralmente no domicílio do profissional.

Tabela 2 - Atualização e uso das bases de dados para buscar PBE (16)

Características

\section{Meios de atualização profissional}

Cursos

Congressos, palestras e conferencias,

Artigos científicos

Frequência das bases de dados nos últimos 6 meses

Todos os dias

De 1 a $3 x$ semana

De 1 A $3 x$ no mês

1 vez a cada 2 meses

Muito raramente

Quais os locais que realiza a busca das bases de dados

Casa

Trabalho

Universidade

Bases de dados que já utilizou

Pubmed

Cochrane

Scielo

Lilacs

PEDro

Google acadêmico

Bases de dados que mais faz o seu uso

Pubmed

Cochrane

Scielo

Lilacs

PEDro

Google acadêmico

\section{n $(\%)$}

$4(25,0)$

$3(18,8)$

$9(56,3)$

$1(6,3)$

$5(31,3)$

$4(25,0)$

$3(18,8)$

$3(18,8)$

$11(68,8)$

$4(25,0)$

$1(6,3)$

SIM n (\%)

$15(93,8)$

$13(81,3)$

$15(93,8)$

$15(93,8)$

$12(75,0)$

$11(68,8)$

$13(81,3)$

$4(25,0)$

$15(93,8)$

$8(50,0)$

$9(56,3)$

$9(56,3)$
NÃO n (\%)

$1(6,3)$

$3(18,8)$

$1(6,3)$

$1(6,3)$

$4(25,0)$

$5(31,3)$

$3(18,8)$

$12(75,0)$

$1(6,3)$

$5(50,0)$

$7(43,8)$

$7(43,8)$

Fonte: Própria do autor.

Conhecimento, habilidades, recursos e opiniões em relação à $\mathrm{PBE}$

A Tabela 3 apresenta os resultados quanto a conhecimento, habilidades, recursos e opiniões em relação à PBE. Em relação ao conhecimento, 87,5\% dos participantes relataram 
que sabem o significado do termo PBE, apesar de que 43,8\% não tiveram experiência em PBE durante sua formação. A maioria dos participantes declarou acreditar ter conhecimento suficiente para aplicabilidade da PBE (37,5\% concordaram parcialmente e $25 \%$ concordaram totalmente) e 62,5\% declararam interesse em aprofundar seus conhecimentos em PBE.

Sobre habilidades e recursos disponíveis, a maioria dos participantes têm o hábito de acessar bases de dados online (50\% concordam parcialmente e 37,5\% concordam totalmente). Seis deles $(37,5 \%)$ relataram facilidade em realizar buscas através de bases de dados. A maioria apontou que sabe avaliar criticamente um artigo científico (43,8\% concordaram parcialmente e $18,8 \%$ concordaram totalmente). Doze participantes (75\%) declararam que procuram implantar a melhor evidência científica na prática clínica.

A maioria dos participantes afirmou questionar o paciente quanto às suas preferências, considerando-as na tomada de decisão (62,5\% concordaram parcialmente e 31,3\% concordaram totalmente). A maioria também declarou informar as opções de tratamento, decidindo-a em conjunto com o paciente (56,3\% concordaram parcialmente e $18,85 \%$ concordaram totalmente).

Quanto às opiniões, a maioria dos participantes declararam acreditar que a PBE é importante para a prática clínica $(87,5 \%)$ e que melhora o atendimento ao paciente $(75 \%)$. Além disso, a maioria apontou que grande parte da tomada de decisão em relação ao plano de tratamento incorpora a PBE (50\% concordaram parcialmente e $43.8 \%$ concordaram totalmente) e que a utilização da melhor evidência possível auxilia na qualidade dos serviços de saúde $(56,3 \%)$. A maioria dos participantes também declararam que a opinião de especialistas da área é o fator mais importante na tomada de decisão (37,5\% concordaram parcialmente e 18,8\% concordaram totalmente).

Tabela 3 - Repostas relacionadas ao conhecimento, habilidade e recursos e opiniões em Prática Baseada em Evidências

\begin{tabular}{lccccc}
\hline \multicolumn{1}{c}{ Conhecimentos } & $\begin{array}{c}\text { Discordo } \\
\text { totalmente }\end{array}$ & $\begin{array}{c}\text { Discordo } \\
\text { parcialmente }\end{array}$ & Neutro & $\begin{array}{c}\text { Concordo } \\
\text { parcialmente }\end{array}$ & $\begin{array}{c}\text { Concordo } \\
\text { totalmente }\end{array}$ \\
\hline $\begin{array}{l}\text { Sei o significado do } \\
\text { termo Prática Baseada } \\
\text { em Evidências (PBE) }\end{array}$ & 0,0 & 0,0 & 0,0 & 12,5 & $\mathbf{8 7 , 5}$ \\
$\begin{array}{l}\text { Não tive experiência } \\
\text { com PBE na minha }\end{array}$ & 31,3 & 6,3 & 12,5 & $\mathbf{4 3 , 8}$ & 6,3 \\
$\begin{array}{l}\text { formação } \\
\text { As informações que }\end{array}$ & 6,3 & $\mathbf{3 1 , 3}$ & 25,0 & $\mathbf{3 1 , 3}$ & $\mathbf{6 , 3}$ \\
$\begin{array}{l}\text { tive na minha } \\
\text { formação sobre PBE } \\
\text { foram suficientes, }\end{array}$ & 18,8 & $\mathbf{3 7 , 5}$ & 25,0 & 18,8 & 0,0 \\
$\begin{array}{l}\text { Não possuo } \\
\text { compreensão sobre os }\end{array}$ & & & & &
\end{tabular}


elementos centrais da

PBE,

Possuo compreensão

6,3

12,5

37,5

43,8

clara sobre a aplicação

dos dados de uma

pesquisa na prática

clínica,

Possuo entendimento a

6,3

6,3

6,3

43,8

37,5

respeito de diferentes

tipos de estudo,

Não possuo

18,8

37,5

25,0

18,8

0,0

entendimento sobre

dados estatísticos,

Acredito ter

6,3

0,0

31,3

37,5

25,0

conhecimento

suficiente para aplicar a PBE,

Não apresento

62,5

18,8

12,5

0,0

interesse em

aprofundar meus

conhecimentos em

PBE,

\begin{tabular}{|c|c|c|c|c|c|}
\hline $\begin{array}{c}\text { Habilidades e } \\
\text { recursos }\end{array}$ & & & & & \\
\hline $\begin{array}{l}\text { Não possuo facilidade } \\
\text { em realizar buscas } \\
\text { através das bases de } \\
\text { dados, }\end{array}$ & 37,5 & 31,3 & 6,3 & 25,0 & 0,0 \\
\hline $\begin{array}{l}\text { Possuo facilidade em } \\
\text { avaliar criticamente } \\
\text { um artigo científico, }\end{array}$ & 6,3 & 18,8 & 12,5 & 43,8 & 18,8 \\
\hline $\begin{array}{l}\text { Possuo hábito de } \\
\text { acessar bases de dados } \\
\text { online, }\end{array}$ & 6,3 & 0,0 & 6,3 & 50,0 & 37,5 \\
\hline $\begin{array}{l}\text { Não possuo incentivo } \\
\text { do meu trabalho para } \\
\text { implantar a PBE, }\end{array}$ & 18,8 & 25,0 & 31,3 & 18,8 & 6,3 \\
\hline $\begin{array}{l}\text { Possuo recursos como } \\
\text { computador e acesso a } \\
\text { internet no local de } \\
\text { trabalho que facilitam } \\
\text { a implantação da PBE, }\end{array}$ & 0,0 & 25,0 & 12,5 & 43,8 & 18,8 \\
\hline $\begin{array}{l}\text { Não realizo discussões } \\
\text { a respeito de PBE no } \\
\text { meu local de trabalho, }\end{array}$ & 12,5 & 37,5 & 25,0 & 12,5 & 12,5 \\
\hline $\begin{array}{l}\text { Questiono o paciente } \\
\text { em relação a suas } \\
\text { preferências e as }\end{array}$ & 0,0 & 0,0 & 6,3 & 62,5 & 31,3 \\
\hline
\end{tabular}


considero na minha tomada de decisão, Informo o paciente suas opções de $\begin{array}{llll}0,0 & 12,5 & \mathbf{5 6}, 3 & 12,5\end{array}$ tratamento e decido com ele a tomada de decisão,

Nunca busco implanta a melhor evidência científica na minha prática clínica

\begin{tabular}{|c|c|c|c|c|c|}
\hline Opiniões & & & & & \\
\hline $\begin{array}{l}\text { A PBE é importante } \\
\text { para a minha prática } \\
\text { clínica, }\end{array}$ & 0,0 & 0,0 & 0,0 & 12,5 & 87,5 \\
\hline $\begin{array}{l}\text { Eu não acredito que a } \\
\text { PBE melhora o } \\
\text { atendimento do } \\
\text { paciente em } \\
\text { fisioterapia, }\end{array}$ & 75,0 & 12,5 & 0,0 & 6,3 & 6,3 \\
\hline $\begin{array}{l}\text { Grande parte da minha } \\
\text { tomada de decisão em } \\
\text { relação ao tratamento } \\
\text { do meu paciente } \\
\text { incorpora a PBE, }\end{array}$ & 0,0 & 0,0 & 6,3 & 50,0 & 43,8 \\
\hline $\begin{array}{l}\text { A opinião de } \\
\text { especialistas da minha } \\
\text { área é o fator mais } \\
\text { importante na minha } \\
\text { tomada de decisão, }\end{array}$ & 12,5 & 12,5 & 18,8 & 37,5 & 18,8 \\
\hline $\begin{array}{l}\text { A utilização da melhor } \\
\text { evidência científica } \\
\text { atual não auxilia na } \\
\text { qualidade dos serviços } \\
\text { de saúde, }\end{array}$ & 56,3 & 25,0 & 6,3 & 6,3 & 6,3 \\
\hline
\end{tabular}

\section{Barreiras enfrentadas em PBE}

A tabela 4 apresenta as barreiras declaradas pelos participantes em relação à PBE. As barreiras mais relatadas pelos participantes foram o idioma dos artigos científicos $(62,5 \%$ concordaram parcialmente e 12,5\% concordaram totalmente), a falta de critérios para qualificar se há evidências no estudo (62,5\% concordaram parcialmente), a dificuldade de obter o artigo na íntegra (37,5\% concordaram parcialmente e 43,8\% concordaram totalmente), a falta de tempo (62,5\% concordaram parcialmente), a dificuldade de entendimento dos dados estatísticos 
(43,8\% concordaram parcialmente e $31,3 \%$ concordaram totalmente), a interpretação dos resultados $(68,8 \%$ concordaram parcialmente) e a falta de treinamento em PBE $(62,5 \%$ concordaram parcialmente).

Tabela 4 - Repostas relacionadas as barreiras enfrentadas em Prática Baseada em Evidências

\begin{tabular}{|c|c|c|c|c|c|}
\hline Barreiras enfrentadas & $\begin{array}{l}\text { Discordo } \\
\text { totalmente }\end{array}$ & $\begin{array}{c}\text { Discordo } \\
\text { parcialmente }\end{array}$ & Neutro & $\begin{array}{c}\text { Concordo } \\
\text { parcialmente }\end{array}$ & $\begin{array}{l}\text { Concordo } \\
\text { totalmente }\end{array}$ \\
\hline $\begin{array}{l}\text { Idioma dos artigos } \\
\text { científicos }\end{array}$ & 0,0 & 6,3 & 18,8 & 62,5 & 12,5 \\
\hline $\begin{array}{l}\text { Falta de qualidade das } \\
\text { evidências }\end{array}$ & 12,5 & 0,0 & 12,5 & 62,5 & 12,5 \\
\hline $\begin{array}{l}\text { Dificuldade de obter o } \\
\text { artigo na íntegra }\end{array}$ & 0,0 & 18,8 & 0,0 & 37,5 & 43,8 \\
\hline Falta de tempo & 0,0 & 12,5 & 25,0 & 62,5 & 0,0 \\
\hline $\begin{array}{l}\text { Entender dados } \\
\text { estatísticos }\end{array}$ & 0,0 & 6,3 & 18,8 & 43,8 & 31,3 \\
\hline $\begin{array}{l}\text { Entender os resultados } \\
\text { (conflitantes) }\end{array}$ & 12,5 & 0,0 & 6,3 & 68,8 & 12,5 \\
\hline $\begin{array}{l}\text { Dificuldade em explicar } \\
\text { para o paciente (nível de } \\
\text { compreensão) }\end{array}$ & 6,3 & 0,0 & 37,5 & 43,8 & 12,5 \\
\hline $\begin{array}{l}\text { Aplicabilidade da } \\
\text { pesquisa na prática } \\
\text { clínica }\end{array}$ & 0,0 & 18,8 & 43,8 & 25,0 & 12,5 \\
\hline $\begin{array}{l}\text { Falta de treinamento em } \\
\text { Prática Baseada em } \\
\text { Evidências }\end{array}$ & 12,5 & 0,0 & 12,5 & 62,5 & 12,5 \\
\hline $\begin{array}{l}\text { Falta de noções básicas } \\
\text { em pesquisa }\end{array}$ & 25,0 & 12,5 & 6,3 & 50,0 & 6,3 \\
\hline $\begin{array}{l}\text { Incapacidade de avaliar a } \\
\text { qualidade do estudo }\end{array}$ & 18,8 & 12,5 & 25,0 & 43,8 & 0,0 \\
\hline $\begin{array}{l}\text { Não implantação da } \\
\text { pesquisa científica }\end{array}$ & 12,5 & 6,3 & 18,8 & 50,0 & 12,5 \\
\hline $\begin{array}{l}\text { Falta de interesse por } \\
\text { pesquisa }\end{array}$ & 31,3 & 18,8 & 37,5 & 12,5 & 0,0 \\
\hline $\begin{array}{l}\text { Acredita que a Prática } \\
\text { Baseada em Evidências } \\
\text { desconsidera as } \\
\text { preferências do paciente }\end{array}$ & 31,3 & 25,0 & 12,5 & 31,3 & 0,0 \\
\hline $\begin{array}{l}\text { Utilizar a Prática } \\
\text { Baseada em Evidências } \\
\text { pode representar maior } \\
\text { custo }\end{array}$ & 56,3 & 18,8 & 12,5 & 12,5 & 0,0 \\
\hline $\begin{array}{l}\text { Desconhecimento sobre } \\
\text { como usar as bases de } \\
\text { dados }\end{array}$ & 18,8 & 25,0 & 25,0 & 31,3 & 0,0 \\
\hline
\end{tabular}




\section{Discussão}

Os resultados desse estudo demonstraram que os participantes costumam atualizar-se através de artigos científicos (56,3\%), sendo que a base mais procurada é a Scielo $(93,8 \%)$ seguida da Pubmed (81,3\%).

Os participantes declararam conhecer o significado do termo PBE e têm conhecimento quanto à sua importância na prática clínica e sua aplicabilidade. Também apresentaram interesse em aprofundar seus conhecimentos sobre o tema, já que 43,8\% relataram que não tiveram experiência em PBE durante sua formação. Esse fato pode ser parcialmente explicado pelo tempo de formação de grande parte dos participantes ser superior a 10 anos, uma vez que este tema de estudo é de popularização recente no Brasil. Também pode ter contribuído com a falta de experiência em PBE, a formação dos participantes majoritariamente em instituição privada $(56,3 \%)$. Uma vez que as instituições superiores públicas concentram quase toda a pesquisa no Brasil, os alunos de instituições privadas não recebem o mesmo incentivo à busca de artigos em bases de dados e ao estudo criterioso da qualidade do artigo pesquisado ${ }^{22}$.

A maioria dos fisioterapeutas participantes desta pesquisa informaram que procuram implantar a melhor evidência científica na prática clínica e questionam o paciente quanto às preferências considerando-as para o plano de tratamento. Por outro lado, relataram que a opinião de especialistas da área é o fator mais importante na sua tomada de decisão, demonstrando dificuldade em utilizar a própria experiência clínica na resolução dos problemas informados pelos pacientes. Isso os distancia de um dos pilares em PBE: experiência clinica do profissional $^{10}$.

As barreiras mais frequentemente encontradas foram: idioma do artigo científico, falta da qualidade das evidências, falta de tempo, escassez de artigos na íntegra, dificuldade em compreender os resultados dos artigos e dados estatísticos e a falta de treinamento em PBE.

Ao nosso ver, a principal contribuição deste estudo é sua abordagem da PBE no ambiente hospitalar. Segundo o levantamento realizado nas bases de dados empregadas, o presente estudo mostra-se precursor no Brasil nessa escolha. Assim, os resultados aqui obtidos poderão contribuir no levantamento de informações sobre PBE e sua relação com a qualidade do trabalho em fisioterapia em hospitais, a quantidade de respondentes foi de $72 \%$ sendo-o adquirido em um tempo de 20 dias após o envio por correio eletrônico.

Como limitação do estudo, a abrangência da população de profissionais fisioterapeutas de atuação hospitalar, neste estudo, é muito pequena, pois esta pesquisa, focou somente em uma instituição de saúde na mesma instituição onde trabalham, logo não se pode garantir que os 
resultados obtidos nesse estudo reflitam a realidade de outros hospitais, publico, mas, principalmente do setor privado.

Estudo de Silva, Costa e Costa realizado com 316 fisioterapeutas brasileiros, residentes no estado de São Paulo, de todas as especialidades, identificou conhecimentos, habilidades, recursos e opiniões em relação à PBE ${ }^{10}$. Assim como em nosso estudo, o perfil demográfico foi de maioria feminina, com formação em instituição privada, com especialização em pósgraduação lato sensu. A maioria também atuava diretamente com o atendimento ao paciente. Além disso, grande parte dos participantes daquele estudo também relatou não ter experiência prévia como docente ${ }^{10}$.

Corroborando com nosso estudo, os fisioterapeutas entrevistados por Silva, Costa e Costa apontaram utilizar artigos científicos para atualização profissional, com preferência do uso de base de dados em português, sendo que também utilizavam preferencialmente a base Scielo. Relataram possuir compreensão sobre a aplicação da PBE na prática clínica, acreditavam ter conhecimento e habilidades para o seu uso, tinham opinião favorável a respeito da PBE e assumiam que seu uso melhora o atendimento ao paciente. Como no nosso estudo, os informantes de Silva, Costa e Costa relataram dificuldade no entendimento dos dados estatísticos. Por outro lado, diferentemente do relatado pelos participantes deste estudo, os informantes daquela pesquisa informaram dificuldades quanto aos elementos centrais da $\mathrm{PBE}^{10}$.

Em relação às barreiras encontradas, similarmente à nossa pesquisa, os informantes de Silva, Costa e Costa consideraram obstáculo relevante o idioma do artigo científico e a dificuldade em obtê-lo na íntegra (10). Por outro lado, acreditam que a PBE pode representar maior custo $^{10}$. Tal dado não ocorre em nosso estudo, mas pode ser entendido devido à dificuldade em acesso gratuito aos artigos disponíveis nas bases de dados mais relevantes.

Claudino, Simões e Silva realizaram estudo com 101 fisioterapeutas brasileiros da área de fisioterapia dermatofuncional ${ }^{2}$. O perfil demográfico foi semelhante ao do nosso estudo com a maioria feminina, com graduação realizada em instituição privada e especialização lato sensu. Grande parte dos participantes trabalhava diretamente com pacientes. Diferentemente de nossa pesquisa, a maioria dos participantes tinham experiência com a docência ${ }^{2}$.

Os informantes de Claudino, Simões e Silva relataram atualizar-se através de cursos e de artigos científicos. As bases de dados por eles mais utilizadas eram em língua portuguesa (Lilacs e Scielo), mas também acessavam frequentemente a Pubmed, assim como os participantes do presente estudo. Reportaram saber o significado da PBE e compreender claramente a aplicação dos achados na prática clínica, além de acreditar ter conhecimento suficiente para sua aplicabilidade. Houve inconsistência nas respostas quanto às informações 
sobre PBE terem sido suficientes. Apesar de acreditarem que a PBE é importante, consideravam a opinião do especialista como principal fator na tomada de decisão clínica ${ }^{2}$. Dessa forma, demonstraram que, por vezes, o que apresenta resultados positivos na prática do dia a dia tem grande relevância para o profissional na tomada de decisão.

As barreiras relatadas pelos informantes de Claudino, Simões e Silva foram o idioma do artigo, a falta da qualidade das evidências, a escassez de artigos na íntegra, a falta de tempo, a dificuldade em compreender os resultados e a aplicabilidade na prática clínica ${ }^{2}$.

Estudo de Queiroz e Santos realizado com 67 fisioterapeutas de todas as especialidades da região da Grande Florianópolis, SC, buscou identificar se os mesmos tinham habilidade e facilidade em realizar pesquisas na literatura cientifica e se utilizavam a PBE na prática clínica. Os resultados demonstraram que os fisioterapeutas apresentavam atitudes positivas quanto à PBE e demonstravam interesse em aprimoramento de suas competências e, consequentemente, em aumentar a quantidade de informações e ferramentas utilizadas em suas práticas clínicas ${ }^{11}$.

A presente pesquisa demonstrou que os fisioterapeutas hospitalares do HRSJ apresentaram atributos (pilares) em relação à PBE. Isso reafirma o caráter científico da profissão e reforça a necessidade de: acesso, aperfeiçoamento profissional através da disponibilização das grandes bases de dados, tanto para o profissional em formação quanto para os egressos enquanto parâmetros que possam nortear a busca pela qualidade de criticidade de estudos clínicos.

Este estudo sinaliza a importância da inclusão dos princípios de PBE, imediatamente, durante a vida acadêmica e que esta ação possa ser fortalecida na formação continuada, por meio de cursos e de acesso a informação científica atual e de qualidade em bases de dados relevantes, além, do incentivo do profissional na busca de informações atuais para oferecer condições de atendimentos conforme os pilares da PBE preconiza. Essas ações gerarão melhorias na qualidade da assistência prestada pela fisioterapia $3,17,18,19$. Também deve ser considerada a possibilidade de que aos conselhos federal, regionais e associações, específicas, para cada especialidade criem mecanismos de atualização em PBE durante cursos de pósgraduação lato sensu. Essas ações poderiam auxiliar na real aplicabilidade dos dados das pesquisas de alta qualidade na resolução de problemas na tomada de decisão clínica ${ }^{20}$.

Nossa pesquisa aponta para a necessidade de estudos futuros que incluam os profissionais das cinco regiões brasileiras que trabalhem na área hospitalar, tanto no setor público quanto privado. Além disso, perante a escassez de estudos brasileiros relacionados à PBE, sugere-se também pesquisa envolvendo fisioterapeutas das diferentes áreas em âmbito nacional. 


\section{Conclusão}

Os fisioterapeutas atuantes no HRSJ relataram atitudes positivas quanto ao uso da PBE, conhecimento suficiente para sua aplicabilidade e interesse em aprofundar seus conhecimentos sobre o tema, a fim de utilizar a melhor evidência científica na prática clínica. Por outro lado, foram relatadas barreiras que dificultam a implantação da PBE na rotina diária de atendimento: dificuldade com o idioma do artigo científico; falta da qualidade das evidências; falta de tempo; escassez de artigos na íntegra; dificuldade em compreender os resultados dos artigos e dados estatísticos; falta de treinamento em PBE. Cabe ressaltar que, apesar de os fisioterapeutas se apresentarem favoráveis à PBE, relataram que a opinião de outro especialistas da área é ainda o fator mais importante na tomada de decisão.

\section{Referências}

1. Silva TM, Garcia NA, Costa LOP. Prática baseada em evidência: como efetivamente utilizar na prática clínica. In: Oliveira GDSC, Macedo RR, editors. Porto Alegre: Artmed; 2016.

2. Claudino R, Simões NP, Silva T. Evidence-Based Practice: a survey of Brazilian physical therapists from the dermatology subdiscipline. Braz J Phys Ther. 2018, [citado 2019 maio 07] http://doi.org/10.1016/j.bjpt.2018.10.002.

3. Silva TM, Costa LCM, Garcia NA, Costa LOP. What do physical therapists think about Evidence-Based Practice? A systematic review. Man Therapy. 2015;20 (3):388-401.

4. Hebert et al. Practical Evidence-Based physiotherapy. 2nd ed. United Kingdom: Butterworth-Heinemann; 2011.

5. Haynes B, Haines A, Barriers and bridges to evidence based clinical practice. BMJ 1998;317 (7153): 273-6.

6. Alves NA. A importância da atuação do fisioterapeuta no ambiente hospitalar. Ensaios e Ciência: Ciências Biológicas, agrárias e da Saúde. 2012;16 (6):173-184.

7. COFFITO. Acórdão no 472, de 20 de maio de 2016 - Dispõe sobre o trabalho do Fisioterapeuta no período de 24 horas em CTIs. Acessado em 19 novembro de 2018.

8. Sarmento, GJV. Fisioterapia respiratória no paciente crítico: rotinas clínicas. 2 ed. São Paulo: Manole; 2007.

9. Sommers J et al. Physiotherapy in the intensive care unit: An evidence-based, expert driven, practical statement and rehabilitation recommendations. Clin Rehabil. 2015;29 (11):1051-63.

10. Silva TM, Costa LC, Costa LO. Evidence-Based Practice: a survey regarding behavior, knowledge, skills, resources, opinions and perceived barriers of Brazilian physical 
therapists from Sao Paulo state. Brazilian journal of physical therapy. 2015;19 (4):294303.

11. Queiroz PS, Santos MJD. Facilidades e habilidades do fisioterapeuta na procura, interpretação e aplicação do conhecimento científico na prática clínica: um estudo piloto easiness and skillfulness of physical therapists in searching, interpreting and applying scientific knowledge in clinical practice: a pilot study. Fisioter Mov. 2013; 26 (1):13-23.

12. Kume J, Tandel R, Indelicato J. Application of the Evidence-Based Practice Model by Physical Therapy Clinical Practitioners. J Allied Health. 2019 Fall;48 (3):e79-e85.

13. Alshehri MA, Alalawi A, Alhasan H, Stokes E. Physiotherapists' behaviour, attitudes, awareness, knowledge and barriers in relation to evidence-based practice implementation in Saudi Arabia: a cross-sectional study. Int J Evid Based Healthc. 2017;15 (3):127-14.

14. Yahui HC, Swaminathan N. Knowledge, attitudes, and barriers towards evidence-based practice among physiotherapists in Malaysia. Hong Kong Physiother J. 2017; 37:10-18.

15. Alrowayeh HN, Buabbas AJ, Alshatti TA, AlSaleh FM, Abulhasan JF. Evidence-Based Physical Therapy Practice in the State of Kuwait: A Survey of Attitudes, Beliefs, Knowledge, Skills, and Barriers. JMIR Med Educ. 2019;5 (1):e12795.

16. Fujimoto S, Kon N, Takasugi J, Nakayama T. Attitudes, knowledge and behavior of Japanese physical therapists with regard to evidence-based practice and clinical practice guidelines: a cross-sectional mail survey. J Phys Ther Sci. 2017;29 (2):198-208.

17. Silva AM, Comper ML, Costa LdCM, Padula RS. Instrumentos para avaliar a prática baseada em evidências na fisioterapia: uma revisão sistemática. Conscientiae saúde (Impr). 2015;14 (2):321-7.

18. Tilson JK. Validation of the modified Fresno test: assessing physical therapists' evidence based practice knowledge and skills. BMC medical education. 2010;10 (1):38.

19. Virtuoso JF, Haupenthal A, Pereira ND, Martins C, Knabben R, Andrade A. A produção de conhecimento em fisioterapia: análise de periódicos nacionais (1996 a 2009).

Fisioterapia em Movimento. 2011;24 (1):173-80.

20. Vinha DRD. Fisioterapia baseada em evidências: uma experiência prática de ensino. Tuiuti: Ciência e Cultura. 2002; 26:87-108.

21. Jang MH, Shin MJ, Shin YB. Pulmonary and Physical Rehabilitation in Critically Ill Patients. Acute Crit Care. 2019;34 (1):1-13.

22. Hilu L, Gisi ML. Produção científica no Brasil - um comparativo entre as universidades públicas e privadas. X Congresso Nacional de Educação - EDUCERE / I Seminário Internacional de Representações Sociais, Subjetividade e Educação - SIRSSE. Anais.... 2011 [citado 2020 maio 16] https://educere.bruc.com.br/CD2011/pdf/5221_3061.pdf. 